Elena Esposito

\title{
Konstruktion der Zukunft und Gebrauch der Zukunft: Risiko als Gelegenheit
}

\section{Zirkularität der Finan₹märte}

Ein paar Jahre nach Ausbruch der Finanzkrise müssen wir zugeben, dass ihre theoretische Auswirkungen bescheiden geblieben sind: Trotz der Dringlichkeit und der Sorge, und trotz einer immer mehr verbreiteten moralischen Alarm, in der Tat operiert man weiterhin aufgrund der selben Kategorien. Die Stimmung hat sich sicher verändert: die «irrationale Euphorie» (gekündigt von Alan Greenspan in 1996) ist von einem wachsenden Ungeduld für die Mechanismen des Marktes ersetzt worden, bis zum Misstrauen in ihren Voraussetzungen. Der eklatanteste und am heftigsten verworfene Aspekt sind natürlich die Belohnungen der Bankiers und die Verhaltensweisen, die als zynisch interpretiert werden. Sie führen dann zum weit verbreiteten obwohl generischen Vorwurf der Gier (oder der übermäBigen Gier - wobei es nicht klar ist, wo der Übermaß anfängt: ob das Problem in der Suche nach Gewinn oder in ihrer Übertreibung liegt).

Im Hintergrund gibt es jedoch einen tieferen und in gewissen Hinsichten mehr beunruhigenden Aspekt: das Bewusstsein, dass die Grundannahmen und die Richtlinien der Finanz unangemessen sind, während noch keine zuverlässige Alternativen zur Verfügung stehen. Trotz aller Bedenken und Debatten über die Reglementierung, und trotz der anhaltenden wirtschaftlichen Krise, haben Banken und Finanzinstitute wieder begonnen, Gewinne zu machen (die Belohnungen sind eigentlich bloß die Folge dieser strukturellen Lage). Wie erklärt man diese Diskrepanz? Es genügt nicht, die Abkopplung zwischen Finanz und Produktion oder zwischen Finanzwirtschaft und Realwirtschaft zu beklagen, solange wir nicht in der Lage sind, zu erklären, wie sie zustande kam noch wovon sie abhängt - oder radikaler, wie sie funktioniert.

Zweifellos hat das Bewusstsein der reflexiven und pro-zyklischen Aspekte des wirtschaftlichen Verhaltens und der Reglementierung selbst zugenommen, bis zu der verbreiteten Paradoxie, dass die Maßnahmen häufig Wirkungen haben, die den Absichten entgegengesetzt sind. Man beginnt zu verstehen, das das Finanzwesen im Guten und im Bösen extrem selbstreferenziell ist und dass man in der Lage sein soll, es zu berücksichtigen. Das ist 
seit langem bekannt. Jetzt scheint es aber immer schwieriger, diese Fragen in einigen Bereichen zu beschränken und zu hoffen, sie unter Kontrolle zu halten: jede Operation und jede Maßnahme werden beobachtet und antizipiert. Das ist ein Teil des dringenden Problems mit dem Ratingverfahren: nicht nur und nicht so sehr der Umstand, die die «Richter» selbst im beurteilten Objekt involviert sind, sondern auch der Umstand, dass die Unternehmer (oder sogar die Staaten) sich für das rating eher als für die Welt vorbereiten. Gute Bewertung zeigt dann immer weniger gute Qualität. Die Zirkularität gehört zu den rating Mechanismen selbst, welche den (nunmehr unumgänglichen) Bezug auf die Welt mit dem Bezug auf die Beobachtung der Welt ersetzen, und dann nicht in der Lage sind, die Auswirkungen zu kontrollieren. Schließlich verstärken die Ratingverfahren genau die Aspekte, die sie unter Kontrolle halten sollten.

Dasselbe Problem betrübt unter der sehr allgemeinen Bezeichnung des moral hazard die Finanzpolitik der Staaten, die beobachtet, antizipiert und neutralisiert werden. Es hilft wenig, es zu wissen: man denke nur an die Dilemmata der sehr umstrittenen Basel 3 Vereinbarung, um die Eigenkapitalanforderungen der Banken zu stärken. Die erklärte und unumstrittene Absicht ist, das Risiko zu verringern, indem die Quote des «zuverlässigen» Kapitals erhöht wird. Das Paradox ist, dass bei Verringerung des Risikos im Banking die Vergütung des Kapitals nachlässt und für die Banken immer schwieriger wird, Geld auf dem Markt zu sammeln - also gerade das Kapital, dass die Banken erhöhen sollten. Jeder stabile Bezug wird instabil im Augenblick, in dem man sich daran wendet.

Zirkularitäten dieser Art befinden sich in allen Aspekten der Finanztätigkeit und verweisen auf die eigentliche Frage, auf das große Rätsel der modernen Finanz: in Finanzmärkten, die eine Masse von Kapital bewegen, die mehr als $20 \mathrm{Mal}$ das gesamte weltweite BIP (Bruttoinlandsprodukt) betragt, was wird tatsächlich gekauft und verkauft? Es ist offensichtlich, dass diese virtuelle Finanz sich nicht auf konkrete Waren und Leistungen bezieht: selbst wenn alle verfügbar wären, würden sie sowieso nicht ausreichen, um die atemberaubenden Zahlen zurückzuzahlen, die auf den Märkten zirkulieren. Worum handelt es sich aber? Was für «Reichtum» wird im hektischen Finanzhandel erstellt oder auch verbrannt?

Obwohl der einfache Verweis auf die Realwirtschaft nicht ausreicht, gibt es natürlich eine Verbindung: der reale Reichtum nimmt zu oder ab nach der finanziellen Transaktionen und ihrem Gang. Sie sind aber nicht Dasselbe: die Finanz hat Auswirkungen auf die Wirtschaft und die Wirtschaft bleibt eine Referenz für die Finanz, aber der Link ist immer indirekter und 
fungibel und läuft vermutlich durch einen weiteren Faktor: die Finanz beeinflusst die Wirtschaft, nicht weil sie realen Reichtum erstellt oder vernichtet, sondern weil sie auf ein anderes Element einwirkt, von dem dieser Reichtum (also die tatsächliche Verfügbarkeit von Waren) abhängt. Worum geht es?

\section{Kauf und Verkauf von Zukunft}

Die Frage ist alt und so ist die Antwort, die mindestens auf Keynes Zeiten zurückreicht. Sie blieb dann wie ein roter Faden im wirtschaftlichen Denken, in der Österreichischen Schule und in einigen post-Keynesianern wie Schackle, aber immer minoritär und fast unterschwellig. Dahinter liegt das Problem des Sinnes und der Funktion von Geld und seiner Verbindung mit der Rolle der Zeit für die Wirtschaft. Was in den Märkten gehandelt wird, ist schließlich Zeit: ihre Verwaltung und ihre Verfügbarkeit in der Gegenwart und vor allem in der Zukunft.

Aus dieser Sicht ist der Bezug auf Zeit, dass die Bedeutung und das Funktionieren der Wirtschaft als Ganzes erklären kann. Geld selbst, das auf der Basis der modernen monetären Wirtschaft liegt, ist eigentlich nichts anderes als Zeit und findet darin seine Funktion (grundsätzlicher als seine Funktion als Tauschmittel, Zahlungsmittel oder Maß des Werts): Geld ist in erster Linie ein «medium of deferment and of search» ${ }^{1}$, das erlaubt, die Befriedigung der Bedürfnisse in einer unbestimmten Zukunft zu verschieben - und dadurch einen Ersatz der Sicherheit anbietet. Vor der obskuren und unerkennbaren Zukunft der Risikogesellschaft ${ }^{2}$ - in der niemand weiß, was man brauchen wird, aber weiß, dass man Bedürfnisse haben wird und ausgerüstet sein möchte, sie zu befriedigen - erlaubt das Besitz von Geld, die Entscheidung zu vertagen und die Informationen zu sammeln, die im Lauf der Zeit entstehen werden. Was auch immer diese Bedürfnisse sein werden (sie müssen nicht heute vorhergesehen werden, noch muss man wissen, wann sie entstehen werden), weiß man, dass wenn man Geld hat, sie befriedigt werden können. Man braucht es nicht, heute die Bedürfnisse von morgen zu kennen. Das Geld steht für die Unbestimmtheit der $\mathrm{Zu}$ kunft: die Zukunft ist noch nicht da und man kann nicht wissen, was man

\footnotetext{
${ }^{1}$ Vgl. Shackle, Time, Expectations and Uncertainty, 213; Epistemic \& Economics, 160.

${ }^{2}$ Vgl. Beck, Die Risikogesellschaft; Luhman, Soziologie des Riskos.
} 
benötigen wird - deshalb braucht man immer Geld, und Geld ist nie genug. Man braucht immer mehr davon, weil auf diese Weise man mehr unbestimmte Möglichkeiten gewinnt, d. h. schließlich mehr Zukunft.

Dieser zeitliche Charakter des Geldes (nicht nur ist Zeit Geld, wie alle sagen, sondern viel radikaler ist Geld Zeit) hat sich auf Finanzmärkten (die bekanntlich direkt Geld verkaufen) enorm verstärkt, vor allem seit den Siebziger Jahren mit der Auflösung des Bretton-Woods-Abkommens, der Verbreitung von Unsicherheit und der Verfügbarkeit von neuen Tools, sie zu behandeln, wie die Modelle für Risikomanagement und vor allem die Explosion der Derivaten. Die geheimnisvolle Bewegung der strukturierte Finanz hat explizit den Bezug auf die Welt und auf konkrete Güter aufgegeben (für Derivate werden die Güter höchstens zum «Basiswert», der alles sein kann und oft in der schwindelnden Transaktion-Verkehr aus den Augen verloren geht), um einen autonomen Bereich von Operationen zu bilden, wo es nicht ganz klar ist, wovon sie handeln.

Die Antwort läuft über zwei Schritte. Zuerst Risiko: wie nunmehr viele sagen $^{3}$, ist die «neue Finanz» der letzten Jahrzehnte vor allem neu, weil jetzt es deutlich geworden ist, dass auf den Märkten direkt Risiko verkauft und weiterverkauft wird - ein abstraktes und formalisiertes, objektiviertes und «commodified» ${ }^{4}$ Risiko, mithilfe von raffinierten Techniken wie die Modelle zur Berechnung und Management der Volatilität ${ }^{5}$. Auf den Märkten wird Volatilität verkauft; Volatilität, welche die Turbulenz und Unvorhersehbarkeit der Märkte misst, steht für Risiko; auf den exoterischen Märkten der strukturierten Finanz, dann, wird in der Tat Risiko verkauft.

Warum aber ist man daran interessiert? Was motiviert diese gigantische Risikozirkulation in der Form von finanziellen Transaktionen? Hier kommen wir zum zweiten Schritt, der zu den Wurzeln der Wirtschaft und des Geldes zurückkommt, also zu unsere Frage der Zeit: in der Form von Risiko kauft man eigentlich die Zukunft, oder möchte man sie kaufen - eine Zukunft, die aus unbestimmten Möglichkeiten, aus offenen Gelegenheiten

${ }^{3}$ Vgl. Arnoldi, Derivatives: Virtual Values and Real Risks; Li Puma/Lee, Financial derivatives and the rise of circulation; Pryke/Allen, Monethized time-space.

${ }^{4} \mathrm{Vgl}$. Bryan/Rafferty, Financial derivatives and the theory of money,136.

5 Vor allem der ganz merkwürdigen «impliziten Volatilität», die mithilfe der Black-Scholes Formel gemessen wird (McKenzie, An Engine, Not a Camera, Kap.5) - ein Verfahren, um die Unvorhersehbarkeit der Zukunft ausgehend von der (nunmehr bekannten) Unvorhersehbarkeit der Vergangenheit zu berechnen. 
besteht, die noch nicht erkannt werden können. Im Verkehr von Risiken kauft und verkauft man die Verfügbarkeit von offenen Möglichkeiten in einer unbekannten Zukunft - die Garantie, dass wenn die Zukunft zur Gegenwart wird, man noch in der Lage sein wird, zu handeln und zu entscheiden. Die Modelle des Portfoliomanagements, mit ihrem Versprechen, in «Risiko-neutralen» Märkten zu operieren, also mit den verschiedenen Risiken und mit Riskantheit im Allgemeinen umgehen zu können, bieten eine Garantie gegenüber Risiken - und tun es auf komplizierter und raffinierter Weise, weil sie nicht beanspruchen, die Zukunft zu kennen. Die Zukunft bleit unbekannt, aber es sollte nicht mehr bedrohlich sein für diejenigen, die Modelle und ihre komplexen Formalisierungen verwenden. Die Berechnung der Volatilität, die Verwendung der Hebelwirkung, von Diversifizierungen und von hoch komplizierte hedging Techniken ${ }^{6}$ tragen dazu bei, Modelle aufzubauen, die versprechen, alle mögliche künftige Entwicklungen zu betrachten - und daher eine Garantie bieten, ohne wissen zu müssen, welcher von diesen Entwicklungen in der Tat auftreten wird. Das Risiko bleibt natürlich, in dem Sinne, dass schädliche Ereignisse sich immer in der Zukunft verwirklichen können und wir heute sie nicht kennen können. Es wird jedoch neutralisiert (nicht gelöscht): für denjenigen, der Modelle verwendet, sollten die gekauften, verkauften und miteinander kombinierten Risiken nicht mehr riskant sein. Was in der Form von Volatilität verkauft wird, ist schließlich die Riskanz des Risikos, d. h. die Offenheit und Verfügbarkeit der Zukunft.

\section{Gegenwärtiger Gebrauch der Zukunft}

Wenn dies zutrifft, oder geglaubt wird, dann macht es Sinn, auch Techniken wie Verbriefungen zu verwenden, die im Grunde Mechanismen sind, um die Zukunft in der Gegenwart zu benutzen: ein künftiger Kredit wird in einem gegenwärtigen Vermögen übersetzt, das dann verwendet wird, um eine Zukunft mit mehr Wohlstand für alle aufzubauen - mit einer Investition, einem Unternehmen, einer jeglichen Tätigkeit, die Liquidität erfordert,

${ }^{6}$ Zusammen mit einer Vielfalt von Techniken, die sich nicht züfällig in denselben Jahren durchgesetzt haben, wie fair value, mark-to-market, Ratingsverfahren - alle Mechanismen, die sich von einer historischen Bewertung (von der Vergangenheit zur Gegenwart) zu einer perspektivischen Einschätzung (von der Zukunft zur Gegenwart) verschoben haben. 
um Gewinne zu erzielen (die es nicht gäbe, wenn der künftige Kredit nicht benutzt wäre). Das hat man in der Wirtschaft seit jeher getan und liegt der Fähigkeit zugrunde, Unsicherheit zu benutzen und die Zukunft als Ressource zu verwenden: alle Formen des Kredits beruhen auf einem Kreislauf von Vorgriff auf die Zukunft in der Gegenwart - man benutz heute das morgigen Geld, um den Reichtum zu produzieren, der morgen (auch) ermöglichen wird, den Schulden zurück zu entrichten. In der jüngsten Finanziarisierung des Kredits wurde aber dieser Mechanismus bis zu seinen extremen Folgen radikalisiert: man beschränkt sich nicht wie in der Vergangenheit auf einen als ziemlich zuverlässig geschätzten künftigen Lauf (wofür Garantien verlangt und Informationen gesucht wurden), sondern verwendet alle mögliche künftige Läufe: all diejenigen, die von den RisikoManagement-Modelle betrachtet werden, welche mit Hilfe der finanziellen Techniken miteinander kombiniert und kompensiert werden. Man braucht nicht einmal, zu viele Garantien über die Solvabilität der Gläubiger abzuholen (selbst NINJA Hypotheken - No Job No Income No Asset - wurden gewährt) weil es geglaubt wird, auf jedem Fall geschützt zu sein, da die Modelle jede Möglichkeit in Betracht ziehen und fähig sind, damit umzugehen. Dann ist es ratsam, und sieht nicht gewagt aus, in der Gegenwart alle diese künftige Verfügbarkeiten zu verwenden, die nicht mehr riskant sind und auf die man sich verlassen kann - wie es mit dem Verkauf und Wiederverkauf von Risiken und Risiken von Risiken im Kreislauf der Verbriefungen, ABS (Asset-Backed-Securities), CDOs (Collateralized-DebtObligations) und CDOs von CDOs und so weiter passiert. Dieser Logik zufolge, sollte das keine Multiplikation der Risiken sondern eine bewusste Konstruktion der Zukunft und ihrer Gelegenheiten sein: indem die Modelle die Zukunft in der Gegenwart antizipieren, versprechen sie, die Vielfalt der ermöglichten Zukünfte zu erhöhen.

Eine oft nicht bewusste Folge dieser Haltung ist, dass der moralische Sinn der Schuld sich ändert. Verschuldung hatte implizit eine Schuldkomponente, auch weil sie gegen die etablierten Idee der Vorsicht (prudentia) ging. Seit Jahrhunderten dachte man, dass Vorsicht darauf beruhte, Risiken zu vermeiden; wer sich entschied, zu riskieren, musste die (auch moralische) Verantwortung dafür tragen - er hatte gewählt, in der Hoffnung eines Gewinns von der Sicherheit abweichen. Der Gewinn konnte aber auch nicht auftreten: in diesem Fall konnte er nicht zu viel mit der Solidarität der Anderen und irgendwie sogar von sich selbst rechnen. Er war selber Schuld. 
In einem vermeintlichen Risiko-neutralen Markt, ändert sich jedoch die Perspektive radikal: Wenn für einen umsichtigen Händler die Unsicherheit der Zukunft keine Bedrohung mehr ist und er immer auf die Füße fallen wird, ist es viel umsichtiger und lobenswerter zu riskieren, anstatt das erworbene Vermögen aufzubewahren. Wer nicht riskiert, ist sogar kleinlich zu sich selbst und zu den Anderen, weil die Tätigkeit auf dem Markt den verfügbare Reichtum für alle erhöht. Die Schuld geht dann eher demjenigen, der sich nicht verschuldet. Vorsicht wird sehr riskant, weil sie bedeutet, auf künftige Gewinne zu verzichten.

Darin lag die Euphorie der neunziger Jahre: der von den Märkten vorausgesetzte Bezug auf die Zukunft war optimistisch und positiv, geleitet vom Vertrauen in der Zunahme der Reichtum wegen der neuen Fähigkeit, Risiko zu verwalten und zu neutralisieren. Beim verbreiteten Misstrauen infolge der Krise sollten wir heute nicht vergessen, dass für einigen Jahren das Modell tatsächlich funktionierte, und dadurch seine Dynamik enorm verstärkte: in der positiven Phase der Finanz vor der Krise, als Kredit für alle zur Verfügung zu stehen schien, haben die Modelle eine Zukunft kompatibel mit ihren Versprechen gebaut - und der finanzielle Wohlstand wuchs für alle. Wie wir aber wissen, hat mit der Krise das Mechanismus gekippt, ist die Zukunft geschlossen und der Reichtum blockiert worden: es scheint, dass es keine offene Möglichkeiten mehr gibt, gerade aufgrund dessen, was wir in der Vergangenheit getan (oder nicht getan) haben. Es stimmt nach wir vor, dass die Modelle die Zukunft gestalten (die ohne sie nicht auf gleicher Weise aufgetreten wäre ${ }^{7}$ ), aber nicht unbedingt die $\mathrm{Zu}$ kunft, die sie vorhergesehen hatten.

\section{Gegenwärtige Zukunft und künftige Gegenwart}

Die Schwäche (wenn man will der Fehler) liegt im Bild der Zeit und der Zukunft dieser Modelle, der rechnerisch sehr kompliziert aber begrifflich zu einfach ist, vor allem in einer komplexen und selbstreferentiellen Gesellschaft wie die heutige und in einem nervösen und reaktiven Bereich wie Finanz. Auch Ökonomen wissen, dass die moderne Gesellschaft sich an

${ }^{7}$ Donald McKenzie spricht in mehreren brillianten und einflussreichen Texten von «Performativität». Cfr. MacKenzie, An Engine, not a Camera; MacKenzie, Is Economics Performative?; MacKenzie, Material Markets; McKenzie/Millo, Constructing a Market, Performing Theory. Der Begriff wurde zuerst in Callon, Introduction, vorgeschlagen. 
eine „offene“ Zukunft orientiert, d.h. eine Zukunft, die nicht vorbestimmt und von oben gegeben ist, sondern radikal unerkennbar ist, weil sie es noch nicht gibt und im Laufe der Zeit entstehen wird. Deshalb orientieren sie ihre Modelle an eine Vielzahl künftiger Zukünfte, wissend, dass niemand heute wissen kann, was morgen auftreten wird. Die Zukunft ist jedoch keinen Vorrat an bereits gegebenen Möglichkeiten, die über Zeit ausgewählt werden, indem einige aktualisiert und andere ausgeschieden werden - wie die Modelle der Risikoverwaltung implizit annehmen, die beanspruchen, alle möglichen Optionen zu betrachten. Wenn es auch gelingen würde, alle Möglichkeiten in Betracht zu ziehen, hätte man sowieso nicht mit der $\mathrm{Zu}$ kunft sonder immer mit der Gegenwart und mit ihren Projektionen zu tun: Was betrachtet wird, ist nur die «gegenwärtige Zukunft» ${ }^{8}$, d.h. das Bild der Zukunft und ihrer Öffnung aus der heutigen Perspektive und aufgrund der zur Zeit verfügbaren Informationen - möglicherweise in Form einer Vielzahl unterschiedlicher gegenwärtigen Zukünfte, die alle mögliche Kombinationen berücksichtigen (aber immer nur diejenige, die gegenwärtig zugänglich sind).

Die Risiko-Management-Modelle orientieren sich an diese Zukunft, die offen bleibt, weil sie in einer Vielzahl verfügbarer künftiger Gegenwarten artikuliert ist, die aber nicht die komplexe und selbstreferenzielle Zukunft ist, welche die aktuellen Risikogesellschaft quält: eine Gesellschaft, die weiß, dass heute die Zukunft nicht als Datum aber auch nicht als Möglichkeit existiert, denn sie wird durch die gegenwärtigen Entscheidungen und Handlungen aufgebaut. Die Möglichkeiten, die künftig verarbeitet und ausgewählt werden, hängen davon ab, was wir heute tun oder nicht tun, in Sicht auf die Zukunft, die wir antizipieren wollen (die aber in der Regel uns überraschen wird). Was künftig real sein wird, ist in der Regel keine der gegenwärtige Zukünfte, sondern eine «künftige Gegenwart»" die anders als jede davon ist, weil sie gerade aus dem Versuch resultiert, die Zukunft vorzubereiten und darauf reagiert. Die einzige Zukunft, dass die Modelle nicht berücksichtigen können, ist diejenige, die sich in der Tat verwirklicht: eine Zukunft, in deren Vergangenheit es die Modelle gibt, die versuchten, sie vorauszusagen. Die Modelle waren nicht falsch (und normalerweise hat man auch während der Krise keine Fehler entdeckt), aber paradoxerweise

\footnotetext{
${ }^{8}$ Vgl. Koselleck, Vergangene Zukunft.

${ }^{9}$ Vgl. Luhmann, Soziologie des Risikos, 48ff.; Esposito, Die Zukunft der Futures, $23 f f$.
} 
haben nicht funktioniert, gerade weil sie korrekt waren und befolgt wurden: Wir könnten sagen, dass sie korrekt alle mögliche künftige Zeitläufe vorsahen, wie sie aufgetreten wären, wenn keine Modelle formuliert würden und dann selbst-falsifizierten sich. Die Zukunft ist umso anders als die Prognose des Modell, je richtiger das Modell ist. Oder genauer gesagt: Wenn die unvorhersehbare Zukunft die Prognosen der Modelle bestätigt, ist es nur Zufall. Es kann passieren oder nicht, aber diese Diskrepanz bildet auf jedem Fall einen Risikofaktor, der die Modelle des Risiko-Managements nicht berücksichtigen können - und dann ist die Welt nicht mehr «Risikoneutral».

\section{Konstruktion der überraschenden Zukunft}

Trotzt allen Revisionen und Veränderungen, können die Modelle diese Zirkularität nicht betrachten - deshalb bleiben die verschiedenen Korrekturen unwirksam und die Märkte operieren weiterhin auf der gleichen Weise. Sie können die unvermeidliche Zirkularität der Orientierung an die Zukunft nicht bewerten - die auch das ist, was die Zukunft immer offen und überraschend macht. Diese Zirkularität ist die Ursache auch des «model risk» produziert vom Gebrauch der Risk-Management-Modelle ${ }^{10}$ und der viel diskutierten «volatility skew», der alle Versuche quält, den Trend der Volatilität mit statistischen Instrumenten zu berechnen ${ }^{11}$. Anders als die Modelle annehmen, folgt der Trend der Volatilität immer häufiger einen pattern (eben den skew oder ironisch den «smile»), der zeigt, dass die Märkte zu erwarten scheinen, dass unwahrscheinliche Ereignisse geschehen werden, während sie als weniger wahrscheinlich schätzen, das wahrscheinliche Läufe eintreten werden. Die Wahrscheinlichkeit wird unwahrscheinlich und die Unwahrscheinlichkeit wahrscheinlich. Mit anderen Worten, es scheint, dass die Märkte gelernt haben, Überraschungen zu erwarten.

Wie erklärt man diese rätselhafte Entwicklung? Das passiert, weil die Märkte beginnen, nicht mehr oder nicht nur die von den Modellen vorweggenommene Zukunft/Zukünfte zu beobachten, sondern selbst die Vorwegnahme der Zukunft durch die Modelle, d.h. die Tatsache, dass Modelle

${ }^{10}$ Vgl. Rebonato, Managing Model Risk.

11 Vgl. MacKenzie/Millo, Constructing a Market, Performing Theory; MacKenzie, An Engine, Not a Camera, 202; Mandelbrot/Hudson, The (mis)Behavior of Markets. 
benutzt und bestimmte Dinge oder Möglichkeiten erwarten werden. Man kann sich dann direkt an dieser Tatsache anstatt an den projizierten $\mathrm{Zu}$ künften wenden und das Unwahrscheinliche erwarten - also das, was die Modelle nicht erwarten. Diese Haltung wird von dem volatility skew registriert, der dazu führt, die Modelle zu falsifizieren und die Risiken zu multiplizieren- nicht die unerwarteten Risiken (die Modelle hatten nichts übersehen) sondern radikaler die unerwartbaren Risiken. Die Märkte werden dann "counter-performative» ${ }^{12}$, also performativ aber grundsätzlich nicht vorhersehbar.

Aus dieser Perspektive erscheint die Finanzkrise als eine Krise der Zukunft: trotzt sorgfältiger Planung und Kontrolle der Investitionen, befinden wir uns in einer Lage, wo wir den Eindruck haben, ohne Zukunft geblieben zu sein - keine gestaltbare Zukunft mehr zur Verfügung zu haben, weil alle Möglichkeiten von den vergangenen Operationen bereits verwendet und gebunden wurden. Der Mangel an Liquidität und die Angst vor Deflation beweisen das: sie sind Phänomene, die aus der Weigerung entstehen, die Zukunft in der Gegenwart zu verwenden - genau das Gegenteil als das, was in den vorigen Jahren getan wurde. Während es früher eine übermäßige Verwendung der Zukunft gab, geht man in der Krise zum entgegengesetzten Übermaß über; so wird die Wirtschaft gelähmt, weil sie wie gesehen gerade auf die Zeit und auf den Aufbau der Zukunft in der Gegenwart beruht.

Die Zukunft tritt dann natürlich sowieso auf, aber unkontrolliert. In einer gewissen Hinsicht stimmt es, dass es weniger Zukunft geben wird, weil weniger Möglichkeiten der Handlung und der Entscheidung generiert werden. Die Zukunft ergibt sich aus der Gegenwart, und das sollte betrachtet werden: Wenn heute nichts getan und nichts geplant wird, wird die Zukunft weniger reich an Gelegenheiten sein, und man wird vermutlich weniger in der Lage sein, sie zu ergreifen und zu nutzen. Das bedeutet jedoch nicht, dass die Zukunft voherrgesehen und bestimmt sein sollte (nach der klassischen Idee von Kontrolle), sondern eher (im kybernetischen Sinne), dass man fähig sein soll, zu verfolgen, wie die heutigen Entscheidungen die (unvorhersehbare) Möglichkeiten generieren, mit denen man in der Zukunft konfrontiert wird. Die Zukunft ergibt sich unvermeidlich aus der Gegen-

12 Cfr. MacKenzie, An Engine, Not a Camera: 184f., 259f.; MacKenzie, Is Economics Performative?, $75 \mathrm{f}$. 
wart - aber genau deshalb ist sie unkontrollierbar und immer überraschend. Die Krise hängt damit zusammen, dass wir nicht gelernt haben, diese Überraschungen zu erwarten und zu verwenden, um unser Verhalten zu leiten wir haben nicht gelernt, die Produktion der Zukunft zu benutzen ohne den Anspruch, ihre Möglichkeiten zu kontrollieren. Luhmann bezeichnet als «Techniken der Defuturisierung» die verschiedenen Versuche, in der Gegenwart die Offenheit und Unkontrollierbarkeit der Zukunft zu binden ${ }^{13}$ : zuerst den gängigen Gebrauch der Statistik, aber auch viele utopische Konstruktionen und natürlich die Moral. Was Märkte heute verlangen, ist nicht die Verwerfung der Technik ${ }^{14}$, also der Verzicht auf den Bau der Zukunft (völlig unnütz, weil die Zukunft sowieso aus unseren Handlungen und Unterlassungen resultiert), sondern vielmehr ein Gebrauch der Technik ohne Defuturisierung, der dagegen anstrebt, die Möglichkeiten zu multiplizieren und zu beobachten - gerade deshalb, weil er nicht beansprucht, sie zu kontrollieren. Diese Art von Haltung ist bereits auf den Märkten vorhanden ${ }^{15}$, noch haben wir aber keine Theorie, die sie beschreibt und überzeugend interpretiert.

\section{Literatur}

Arnoldi, Jakob, Derivatives: Virtual Values and Real Risks, Theory, Culture \& Society, 21, 2004, 23-42.

Beck, Ulrich, Die Risikogesellschaft: Auf dem Weg in eine andere Moderne, Frankfurt a.M., Suhrkamp, 1986.

Bryan, Dick/Rafferty, Michael, Financial derivatives and the theory of money, Economy and Society, 36, 2007, 134-158.

13 Vgl. Luhmann, The Future Cannot Begin.

${ }^{14}$ In der Form von Taleb, Fooled by Randomness.

15 Z. B. die Fälle beschrieben in Preda, Where do analysts come from?, oder die Praxis der «Reflexivität» in Soros, The Alchemy of Finance; siehe auch Esposito, Die Fiktion der wahrscheinlichen Realität, Kap. 13. 
Callon, Michel, Introduction: The embeddedness of economic markets in economics, in: M.Callon, Hg., The Laws of the Market, Oxford, Blackwell, 1998, 1-57.

Esposito, Elena, Die Fiktion der wahrscheinlichen Realität, Frankfurft a.M., Suhrkamp, 2007.

Esposito, Elena, Die Zukunft der Futures. Die Zeit des Geldes im Finanzwesen und in der Gesellschaft, Heidelberg, Auer, 2010.

MacKenzie, Donald, An Engine, Not a Camera. How Financial Models Shape Markets, Cambridge (Mass.), The MIT Press, 2006.

MacKenzie, Donald, Is Economics Performative? Option Theory and the Construction of Derivatives Markets, in: D.McKenzie/F.Muniesa/L.Siu, Hg., Do Economists make Markets?, Princeton, Princeton University Press, 2007, 54-86.

MacKenzie, Donald, Material Markets. How Economic Agents Are Constructed, Oxford: Oxford University Press, 2009.

MacKenzie, Donald/Millo, Yuval, Constructing a Market, Performing Theory: The Historical Sociology of a Financial Derivatives Exchange, American Journal of Sociology, 109, 2003, 107-145.

Koselleck, Reinhart, Vergangene Zukunft. Zur Semantik geschichtlicher Zeiten, Frankfurt a.M., Suhrkamp, 1979.

LiPuma, Edward/Lee, Benjamin, Financial derivatives and the rise of circulation, Economy and Society, 34, 2005, 404-427.

Luhmann, Niklas, The Future Cannot Begin: Temporal Structures in Modern Society, Social Research 43, 1976, 130-152.

Luhmann, Niklas, Soziologie des Risikos, Berlin/New York, de Gruyter, 1991.

Mandelbrot, Benoit/ Hudson, Richard L., The (mis)Behavior of Markets. A Fractal View of Risk, Ruin, and Reward, New York, Basic Books, 2004.

Preda, Alex, Where do analysts come from? The case of financial chartism, in: M.Callon/Y.Millo/F. Muniesa, Hg., Market Devices, Oxford, Blackwell, 2007, 40-64.

Pryke, Michael/Allen, John, Monethized time-space: derivatives - money's 'new imaginary'?, Economy and Society, 29, 2000, 264-284.

Rebonato, Riccardo, Managing Model Risk, in: C. Alexander, Hg., Handbook of Risk Management, Prentice Hall, FT, 2001.

Shackle, George Lennox Sherman, Epistemic \& Economics. A critique of economic doctrins, Cambridge, Cambridge U.P., 1972.

Shackle, George Lennox Sherman, Time, Expectations and Uncertainty in Economics, edited by James Lorne Ford, Aldershot (England), Edward Elgar, 1990. 
Soros, George, The Alchemy of Finance. Reading the Mind of the Market, New York, Wiley, 1987.

Taleb, Nassim Nicholas, Fooled by Randomness. The Hidden Role of Chance in the Markets and in Life, New York-London, Texere LLC, 2001. 